\title{
PERCEPCIÓN CIUDADANA DEL USO DE LAS TIC Y LA ENSEÑANZA ONLINE DURANTE LA PANDEMIA
}

\author{
Patricia González Elices \\ Facultad de Ciencias de la Salud y Educación \\ Universidad a distancia de Madrid, Madrid, España \\ patricia.gonzalez@udima.es
}

Recepción Artículo: 17 mayo 2021 Admisión Evaluación: 17 mayo 2021 Informe Evaluador 1: 22 mayo 2021 Informe Evaluador 2: 25 mayo 2021 Aprobación Publicación: 02 junio 2021

\section{RESUMEN}

La Pandemia que en la actualidad estamos sufriendo a nivel mundial ha provocado un cambio conductual en todos los ámbitos de la vida: restricciones en movilidad, uso obligatorio de mascarillas, etc. Uno de los sectores más afectados ha sido la educación, ya que durante varios meses los centros educativos han estado cerrados, de tal forma que los estudiantes no podían asistir a clase. Pasados los primeros días los centros buscaron implantar como alternativa las clases a distancia a través de plataformas digitales o videoconferencias, no obstante, esta situación puede poner en evidencia e, incluso, ampliar la brecha digital entre algunos estudiantes, aumentando las dificultades de aflabetización debido a que todas las familias no disponen de las mismas oportunidades para acceder a los soportes técnicos necesarios. Dado que la educación es un servicio social y público, se elaboro un formulario con el objetivo de conocer la opinión de la ciudadanía no docente respecto a esta y otras cuestiones relacionadas con las las Tecnologías de la Información y la Comunicación (TIC) para la alfabetización. El cuestionario estaba dividido en cuatro secciones. La primera para recoger datos sociodemográficos básicos e información sobre su situación durante el confinamiento. La segunda donde se preguntaban cuestiones relacionadas con la decisión de hacer online las clases, relacionadas con la brecha digital, la vulnerabilidad, las oportunidades de alfabetización o la competencia digital. La tercera para preguntar por el recurso que consideraban más adecuado y, la cuarta, donde se preguntaba el grado de adecuación de determinadas herramientas durante las clases online. La muestra estuvo compuesta por 47 participantes de entre 25 y 56 años, 30 mujeres y 17 hombres, todos de nacionalidad española aunque de 7 Comunidades Autónomas diferentes. Los resultados indican que la opinión mayoritaria es que los alumnos no han aprendido durante las clases online igual que si hubiesen estado en presencialidad (85,11\%), aunque un 68,09\% señalan que este tipo de clases ha permitido la alfabetización de los estudiantes de Infantil y Primaria. En relación con la brecha digital, un 82,98\% de los participantes señalan que el cierre de los centros educativos la ha incrementado, al igual que la vulnerabilidad de los estudiantes (70,21\%), 


\section{PERCEPCIÓN CIUDADANA DEL USO DE LAS TIC Y LA ENSEÑANZA ONLINE DURANTE LA PANDEMIA}

señalando además, que la utilización de las TIC no promueve las mismas oportunidades de aprendizaje (78,72\%). Los resultados señalan también que las principales herramientas utilizadas para la docencia online están bien valoradas, siendo el uso de plataformas de alojamiento de vídeos como Youtube la menos apreciada. Bajo este prisma y, en consecuencia con el Objetivo de Desarrollo Sostenible 4 (ODS 4) de la Agenda 2030 de la UNESCO que se centra en la garantía de promover las mismas oportunidades de aprendizaje para todos, se concluye que si bien las TIC han permitido abordar de forma temporal una crisis educativa se debe seguir trabajando para que esta alternativa sea un modo realmente efectivo de seguir con la alfabetización en los casos en que la educación obligatoria no pueda realizarse en el centro escolar.

Palabras clave: aprendizaje online; alfabetización; educación; Covid-19; brecha digital

\section{ABSTRACT}

Citizen perception of ict use and e-learning during the pandemic. The pandemic that we are currently suffering worldwide has caused a behavioral change in all areas of life: restrictions on mobility, mandatory use of masks, etc. One of the most affected sectors has been education, since for several months schools have been closed, so that students could not attend classes. After the first days, the centers sought to implement as an alternative distance classes through digital platforms or videoconferencing, however, this situation may highlight and even widen the digital divide among some students, increasing the difficulties of illiteracy because all families do not have the same opportunities to access the necessary technical support. Given that education is a social and public service, a form was developed with the aim of finding out the opinion of nonteaching citizens on this and other issues related to Information and Communication Technologies (ICT) for literacy. The questionnaire was divided into four sections. The first to collect basic socio-demographic data and information about their situation during confinement. The second section asked questions related to the decision to take classes online, related to the digital divide, vulnerability, literacy opportunities or digital competence. The third asked about the resource they considered most appropriate, and the fourth asked about the degree of appropriateness of certain tools during online classes. The sample consisted of 47 participants between 25 and 56 years of age, 30 women and 17 men, all of Spanish nationality although from 7 different Autonomous Communities. The results indicate that the majority opinion is that students have not learned during the online classes as if they had been in the classroom (85.11\%), although 68.09\% say that this type of class has allowed the literacy of students in kindergarten and primary school. In relation to the digital divide, $82.98 \%$ of the participants indicated that the closure of educational centers has increased it, as well as the vulnerability of students $(70.21 \%)$, and that the use of ICTs does not promote equal learning opportunities (78.72\%). The results also indicate that the main tools used for online teaching are well valued, with the use of video hosting platforms such as YouTube being the least appreciated. From this perspective, and in line with Sustainable Development Goal 4 (SDG 4) of UNESCO's 2030 Agenda, which focuses on guaranteeing equal learning opportunities for all, it is concluded that although ICTs have temporarily addressed an educational crisis, more work is needed to ensure that this alternative is a truly effective way of continuing with literacy in cases where compulsory education cannot be carried out in the school center.

Keywords: online learning; literacy; education; Covid-19; digital divide

\section{INTRODUCCIÓN}

La Pandemia que en la actualidad estamos sufriendo a nivel mundial ha provocado un cambio conductual en prácticamente todos los ámbitos de nuestra vida, restricciones en movilidad, uso obligatorio de mascarillas, etc., provocando situaciones excepcionales en determinados sectores. En este contexto es necesario comprender los potenciales cambios derivados del Covid-19. El ámbito educativo ha sido uno de los sectores más afectados pues debido al aumento de contagios y como medida de contención el Gobierno Central decretó un confinamiento domiciliario que provocó el cese de la actividad lectiva presencial. Pasada la primera quincena y observando que, desgraciadamente los contagios no disminuían, este confinamiento tuvo que alargarse, de tal forma que los estu- 
diantes seguían sin poder asistir a clase. Desde el Ministerio de Educación y los propios centros educativos se buscaron alternativas para poder continuar la formación escolar y, estando en casa sin poder salir, la mejor opción (o la única viable) fue modificar el tipo de enseñanza para que las clases, hasta entonces presenciales, pasaran a ser a distancia. Las propias Consejerías de Educación de las diferentes Comunidades Autónomas pusieron a disposición de los docentes diversos recursos, a los que habría que sumar otros software libres que permitieron este sistema de enseñanza. Sin embargo, este entramado de recursos tecnológicos que permiten un sistema de enseñanza online, en cierto sentido pudo crear ciertas dificultades para atender a todo el alumnado. Según el último informe PISA (García, Rivero y Ricis, 2020) un 44\% de las familias más desaventajadas tiene solo un ordenador en casa e incluso un 14\% no tiene ninguno. Esto dificulta el poder ofrecer igualdad de oportunidades ya que no todas las familias disponen del soporte técnico necesario ni el mismo acceso a ellos.

La necesidad de rescindir las clases presenciales y la alternativa de seguir con la enseñanza a través de TIC e Internet condicionan el acceso a una educación equitativa y pueden suponer una mayor brecha digital (término acuñado en los años noventa que refiere la desigualdad en el acceso a estos recursos motivado por las condiciones socioeconómicas y culturales; Gómez, 2019). En este sentido, la literatura científica nos muestra que esta brecha digital en países desarrollados en muchas ocasiones se ve disminuida por los propios usuarios quienes, motivados por su uso, se desplazan buscando alternativas que les permitan conectarse (DiMaggio et al., 2001, citado en Torres, 2017). Ahora bien, ¿qué ocurre con estos usuarios si ven limitada su movilidad? La pandemia supuso no solo los cierres de las instituciones sino la obligatoriedad de un confinamiento domiciliario que impedía desplazarse en busca de Internet o dispositivos tecnológicos. Este confinamiento estaría marcado también por las condiciones materiales de accesibilidad digital para aquellos usuarios que, pese a tener acceso a los dispositivos y a la red viesen dificultado su acceso por la calidad de la conexión y dispositivos o software poco actualizados u obsoletos.

El estudio de Gómez (2019), basado en la Encuesta anual del Instituto Nacional de Estadística (I.N.E.) sobre Equipamiento y Uso de TIC reveló que en 2015 la media de los hogares conectados en España era del 71,3\% y que, en comparación con los años anteriores, el avance de la conexión fija a Internet, redes de banda ancha (ADSL) y el despegue de la fibra óptica se había disparado, por lo que la brecha digital y las desigualdades en Ios jóvenes se debía en mayor medida a la calidad de acceso a Internet. Por tanto, el problema no estaría en tener o no Internet en casa sino en los tipos de dispositivos y, en el tipo de red contratada y la calidad de la señal. Así, familias con menores recursos socioeconómicos tendrían acceso a los servicios TIC pero a los más baratos (conclusiones respaldadas por los datos del I.N.E. 2019). En esta línea, la investigación de Díez y Gajardo (2020) en relación a las condiciones de los hogares españoles durante el confinamiento revela que si bien el 96,2\% puede acceder al menos a un ordenador en su casa, solo un 68\% puede acceder a una conexión de Internet rápida.

Otros estudios señalan además que la brecha digital hoy en día se debe principalmente a las diferencias en la competencia digital de cada usuario (Hargittai, 2002) de tal forma que el cierre de los centros educativos podría haber agravado este aspecto, pues tal y como señala Álvarez-Sigüenza (2019) el hecho de que los estudiantes sean nativos digitales no parece que sea condición suficiente para que tengan las competencias tecnológicas necesarias para poder atender las demandas educativas. Si a ello le sumamos que los propios estudiantes (aproximadamente un 30\% según el estudio de Rodicio-García, Ríos-de Deus, Mosquera-González y Penado, 2020) señalan que no consideraban que durante el confinamiento contaran con recursos tecnológicos suficientes para seguir con sus estudios de manera no presencial, se podría pensar que el cierre de centros ha podido influir en aumentar la desigualdad en la enseñanza escolar, poniendo evidencia e, incluso, ampliando la brecha digital y aumentando las dificultades de aflabetización, ya que, como se ha indicado, todas las familias no disponen de las mismas oportunidades para acceder a los diferentes soportes técnicos que se necesitan para seguir este tipo de clases (García, et al., 2020).

Así mismo, la enseñanza online no solo requiere que los estudiantes tengan ciertas competencias digitales sino que los propios docentes, encargados de trasladar hacia la virtualidad sus clases, necesitan tener ciertas 


\section{PERCEPCIÓN CIUDADANA DEL USO DE LAS TIC Y LA ENSEÑANZA ONLINE DURANTE LA PANDEMIA}

habilidades y tecnología suficiente para poder impartir docencia desde sus casas. La necesidad de llevar las clases presenciales a clases remotas ha supuesto por tanto que los roles de docentes y discentes cambien. Así el estudiante debe empoderarse de su propio proceso de aprendizaje y el profesor debe orientar y confiar en su autonomía (Sierra, 2013; Vellegal, 2009, citado en Pastran, Gil y Cervantes, 2020). En este sentido, si la enseñanza se traslada al hogar, el proceso de enseñanza además recae sobre las familias que están con esos discentes ya que son los encargados de asegurar ese empoderamiento y les ayudan cuando su edad condiciona su nivel de autonomía.

Diversos estudios han analizado las posibilidades y efectividad de la enseñanza desde casa pero siempre desde el prisma de estudiantes o profesores dejando un tanto desatendida la percepción y opinión de las familias, uno de los agentes educativos clave, motivo principal por el que se plantea este pequeño estudio que pretende tomar como punto de vista una opinión global de la ciudadanía no docente, pues no hay que olvidar que la educación es un servicio social y público (López, 2018).

\section{OBJETIVOS}

El objetivo principal de la investigación era analizar la percepción de personal no docente sobre la decisión de trasladar la enseñanza presencial a enseñanza a distancia en base a su opinión sobre los recursos utilizados para tal fin y su valoración sobre las oportunidades que ha brindado. De forma más concreta se establecen los siguientes objetivos: demia.

a) Analizar la percepción sobre la medida de utilizar las TIC para continuar con la enseñanza durante la pan-

b) Conocer qué recurso educativo, usado durante las clases online, es el valorado como más adecuado para las etapas de Educación Infantil y Primaria.

c) Conocer la valoración de la ciudadanía no docente sobre la adecuación de las herramientas utilizadas para la docencia online.

d) Analizar si las competencias digitales se valoran como condicionante de este tipo de clases.

e) Analizar si la enseñanza a distancia se percibe como condicionante de la alfabetización escolar.

f) Analizar si la población no docente considera que el cierre de los centros educativos influye en las oportunidades de aprendizaje e incremento de las desigualdades (brecha digital).

\section{MÉTODO}

\section{Diseño y participantes}

La presente investigación cuantitativa obedece a un diseño no experimental de tipo descriptivo y correlacional (Cohen, Manion y Morrison, 2010). El grado de participación fue algo escaso, no obstante, creemos que suficiente para poder considerar algunas ideas generales. El cuestionario fue respondido por 54 personas, no obstante, 7 señalaron haber estado impartiendo clases online a las etapas de Infantil y Primaria durante el confinamiento, aspecto que hizo que se excluyesen de los análisis. La muestra, por tanto, estuvo compuesta por 47 participantes de entre 25 y 56 años ( $M=40,87), 30$ mujeres (63,83\%) y 17 hombres (36,17\%), todos de nacionalidad española, aunque de 7 Comunidades Autónomas diferentes: 15 de la Comunidad de Madrid (31,91\%), 10 de Cataluña (21,28\%), 8 de la Comunidad Valenciana (17,02\%), 7 de Andalucía (14,89\%), 4 de Extremadura $(8,51 \%)$ y 3 de Asturias $(6,38 \%)$.

Dentro de los 47 participantes $26(55,32 \%)$ indicaron no tener menores de edad a su cargo durante el confinamiento. Por su parte, del $41,68 \%$ restante, 17 personas indicaron estar cuidando a sus hijos (36,17\%) y 4 personas comunicaron que tuvieron a su cargo a menores no familiares, como vecinos o sobrinos (8,51\%).

Finalmente, 28 sujetos $(59,57 \%)$ señalaron no trabajar fuera de casa durante el confinamiento mientras que 19 (40,43\%) sí lo hacían. 


\section{Procedimiento}

La obtención de datos se realizó a través de la técnica de "Bola de nieve", método no discriminatorio exponencial. Para tal fin se utilizaron diferentes RRSS (como Facebook o Linkedln) a través de las cuales se lanzaba el enlace del cuestionario solicitando la colaboración directa y la redistribución del link a conocidos. En la cabecera del cuestionario se informaba de los objetivos del estudio, así como una alusión a la la Ley Orgánica 3/2018, de 5 de diciembre, de Protección de Datos Personales y garantía de los derechos digitales.

El cuestionario se elaboró utilizando la herramienta gratuita para la creación de formularios Google Forms. Esta herramienta facilita una hoja de cálculos con los datos recogidos. A raíz de este documento se elaboró una base de datos para el sistema de software estadístico SPSS (del inglés Statistical Package for Social Sciences), permitiendo así analizar los datos y obtener los resultados que se muestran en el apartado correspondiente.

\section{Instrumentos y variables}

Como se ha indicado para la investigación se utilizó un cuestionario elaborado ad hoc (véase el anexo 1). El cuestionario se dividió en cuatro secciones. Tras la presentación en la cabecera se preguntaba, en la primera sección, datos sociodemográficos básicos sobre la edad, el sexo, y la Comunidad Autónoma de los participantes, así como unas preguntas básicas sobre su situación durante el confinamiento (principalmente personas al cargo durante el confinamiento y trabajo como docente de las etapas de Educación Infantil o Primaria). La segunda sección estaba compuesta por 10 afirmaciones relacionadas con la alfabetización durante el confinamiento que debían ser contestadas a través de una escala dicotómica "si/no". Por su parte, la tercera sección estaba destinada a conocer el recurso que entre la población no docente era mejor valorado para llevar a cabo las clases online, dividiendo esta cuestión en dos, para preguntar de forma independiente las etapas de Infantil y Primaria. Finalmente, la cuarta sección preguntaba el grado de adecuación de algunas herramientas que han sido utilizadas durante las clases online llevadas a cabo en el confinamiento domiciliario.

\section{Análisis de datos}

Para el análisis de las variables se utilizó el programa estadístico SPSS en su versión 22.0. Las variables sociodemográficas se analizaron haciendo uso de frecuencias y descriptivos. Así mismo, las variables dicotómicas o las preguntas de elección única se analizaron, además de con frecuencias a través de tablas de contingencia.

\section{RESULTADOS}

Los resultados de la sección primera del cuestionario pueden observarse en la Tabla 1. Como aspectos destacables indicar que un 82,98\% indicó que la modalidad online a través de las TIC era la única forma de que los alumnos continuasen con la enseñanza durante el confinamiento. No obstante, un $85,11 \%$ señala que no consideran que los estudiantes no han aprendido con estas clases igual que si hubiesen acudido de forma presencial. En esta línea, el 68,09\% señalan que este tipo de clases, mediadas por las TIC, han permitido la alfabetización de los estudiantes de Infantil y Primaria. Destacan, en contraste, la opinión mayoritaria $(82,98 \%)$ de que el cierre de los centros educativos y la docencia online ha incrementado la brecha digital. Únicamente el 21,28\% considera que este tipo de enseñanza ofrece las mismas oportunidades a los estudiantes e incluso el 70,21\% opinan que aumenta la vulnerabilidad de los alumnos menores de edad.

Los resultados muestran que una de las variables más importantes en este tipo de educación es la competencia digital. En este sentido, el 78,72\% de los encuestados señalan que la alfabetización que tuvo lugar durante el confinamiento estaba condicionada por las competencias digitales que tuviesen los estudiantes, un $87,23 \%$ por la que tuviesen los docentes y un $82,98 \%$ por las habilidades o conocimientos de las TIC que tuviesen los tutores/madres o padres. 
Tabla 1. Opinión sobre la alfabetización durante el confinamiento

\begin{tabular}{|c|c|c|}
\hline Afirmación & $\begin{array}{c}\text { Escala de } \\
\text { valor }\end{array}$ & Valor (\% ) \\
\hline \multirow{2}{*}{$\begin{array}{l}\text { Considero que la utilización de las TIC } \\
\text { para continuar con la enseñanza (en } \\
\text { modalidad online) era lo único que se } \\
\text { podía hacer }\end{array}$} & Sí & $39(82,98 \%)$ \\
\hline & No & $8(17,02 \%)$ \\
\hline \multirow{2}{*}{$\begin{array}{l}\text { Considero que los estudiantes han } \\
\text { aprendido lo mismo con la enseñanza } \\
\text { online que si hubiesen acudido a clase de } \\
\text { forma presencial }\end{array}$} & Sí & $7(14,89 \%)$ \\
\hline & No & $40(85,11 \%)$ \\
\hline \multirow{2}{*}{$\begin{array}{l}\text { Considero que la utilización de las TIC } \\
\text { para continuar con la enseñanza (en } \\
\text { modalidad online) promueve las mismas } \\
\text { oportunidades de aprendizaje para todos } \\
\text { los estudiantes) }\end{array}$} & Sí & $10(21,28 \%)$ \\
\hline & No & $37(78,72 \%)$ \\
\hline \multirow{2}{*}{$\begin{array}{l}\text { Considero que el cierre de los centros } \\
\text { educativos ha incrementado la brecha } \\
\text { digital entre los estudiantes (desigualdad } \\
\text { en el acceso a Internet, a los dispositivos } \\
\text { digitales, etc.) }\end{array}$} & Sí & $39(82,98 \%)$ \\
\hline & No & $8(17,02 \%)$ \\
\hline \multirow{2}{*}{$\begin{array}{l}\text { Considero que el cierre de los centros } \\
\text { educativos aumenta la vulnerabilidad de } \\
\text { los estudiantes (al ser menores de edad) }\end{array}$} & Sí & $33(70,21 \%)$ \\
\hline & No & $14(29,79 \%)$ \\
\hline \multirow{2}{*}{$\begin{array}{l}\text { Considero que los conocimientos que los } \\
\text { tutores, padres o supervisores de los } \\
\text { estudiantes pueden tener del uso de las } \\
\text { TIC puede condicionar la efectividad de } \\
\text { las clases online }\end{array}$} & Sí & $39(82,98 \%)$ \\
\hline & No & $8(17,02 \%)$ \\
\hline \multirow{2}{*}{$\begin{array}{l}\text { Considero que la impartición de clases } \\
\text { online ha permitido la alfabetización de } \\
\text { los estudiantes de Educación Infantil y } \\
\text { Primaria }\end{array}$} & Sí & $32(68,09 \%)$ \\
\hline & No & $15(31,91 \%)$ \\
\hline \multirow{2}{*}{$\begin{array}{l}\text { Considero que la alfabetización de los } \\
\text { estudiantes durante el confinamiento ha } \\
\text { estado condicionada por el nivel de } \\
\text { competencia digital que tuviese el docente }\end{array}$} & Sí & $41(87,23 \%)$ \\
\hline & No & $6(12,77 \%)$ \\
\hline \multirow{2}{*}{$\begin{array}{l}\text { Considero que la alfabetización de los } \\
\text { estudiantes durante el confinamiento ha } \\
\text { estado condicionada por el nivel de } \\
\text { competencia digital que tuvieran los } \\
\text { estudiantes }\end{array}$} & Sí & $37(78,72 \%)$ \\
\hline & No & $10(21,28 \%)$ \\
\hline \multirow{2}{*}{$\begin{array}{l}\text { Considero que con las clases online la } \\
\text { responsabilidad educativa recayó sobre } \\
\text { las familias }\end{array}$} & Sí & $41(87,23 \%)$ \\
\hline & No & $6(12,77 \%)$ \\
\hline
\end{tabular}


En otro orden de ideas, al preguntar por los recursos que los ciudadanos no docentes consideran más propicios para una enseñanza online los resultados muestran el recurso que se considera más apropiado para la etapa de Educación Infantil el uso de juegos educativos (36,17\%) seguido de los videos educativos o video-lecciones (23,40\%). Para la etapa de Educación Primaria, Ios resultados muestran una clara preferencia de la videoconferencia con clases en directo $(48,94 \%$ ) sobre el resto de recursos, llamando la atención que ninguno de los participantes indicó como el recurso más adecuado ni el uso de juegos o videojuegos educativos ni el uso de blogs o Redes Sociales educativas (Tabla 2).

Tabla 2. Recurso mejor valorados para las clases online según etapa educativa

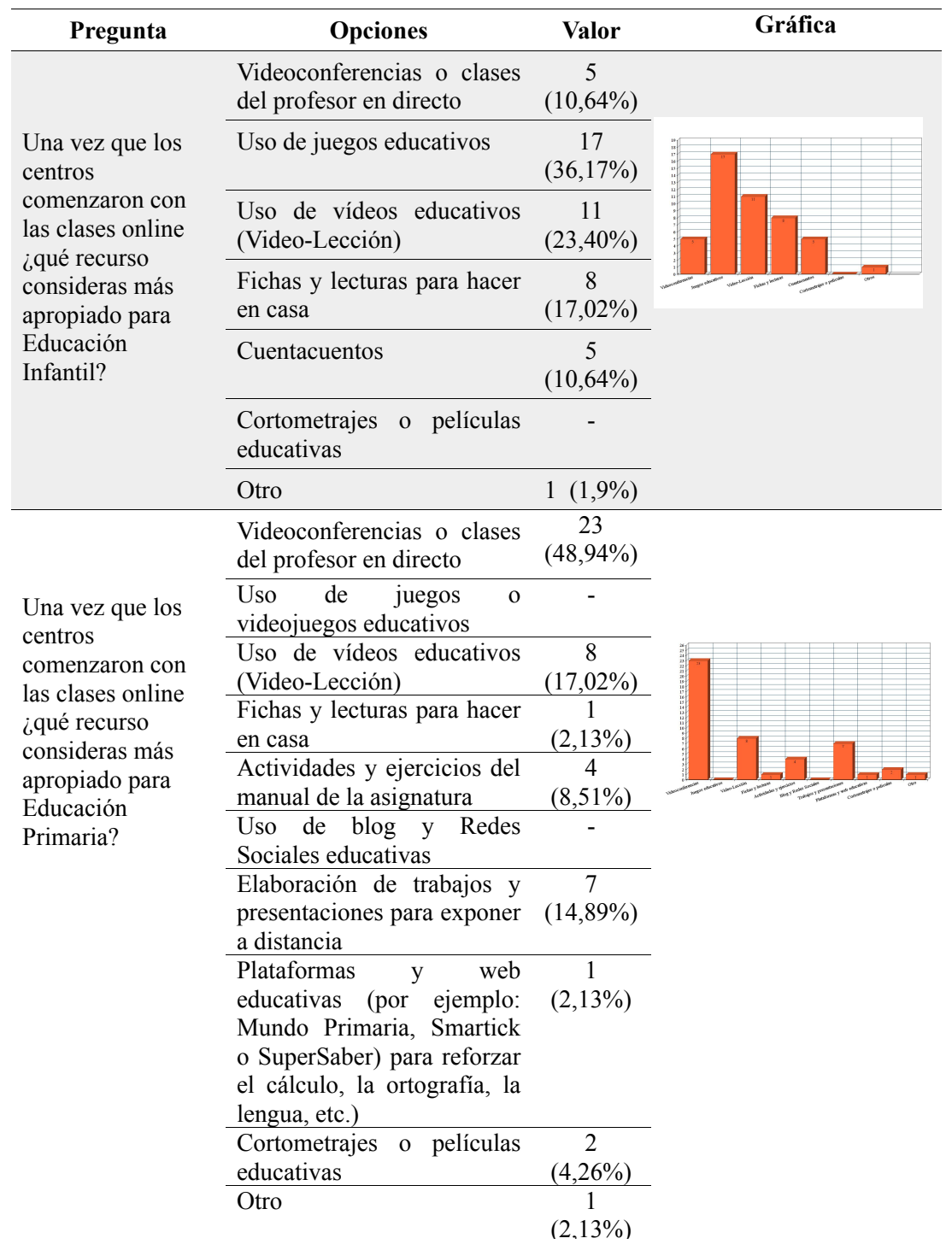




\section{PERCEPCIÓN CIUDADANA DEL USO DE LAS TIC Y LA ENSEÑANZA ONLINE DURANTE LA PANDEMIA}

Finalmente, 4 de las 7 herramientas digitales que se proponían se consideraron adecuadas para la enseñanza online. (Tabla 3). Destaca, no obstante, que aproximadamente la mitad de la muestra $(51,06 \%)$ señala como poco adecuado el uso de plataformas de alojamiento de vídeos. Así mismo, las Redes Sociales educativas o los audiolibros o podcast también muestran algunas valoraciones negativas: $36,17 \%$ y $27,66 \%$, respectivamente.

Tabla 3. Adecuación de las herramientas digitales utilizadas durante el confinamiento

\begin{tabular}{|c|c|c|}
\hline Afirmación & $\begin{array}{l}\text { Escala de } \\
\text { valor }\end{array}$ & Valor (\%) \\
\hline \multirow{2}{*}{$\begin{array}{l}\text { Herramientas de videoconferencias como } \\
\text { Zoom }\end{array}$} & Adecuada & $38(80,85 \%)$ \\
\hline & $\begin{array}{l}\text { No } \\
\text { adecuada }\end{array}$ & $9(19,15 \%)$ \\
\hline \multirow{2}{*}{$\begin{array}{l}\text { Programas para la elaboración de } \\
\text { presentaciones como Genial.ly o Prezi }\end{array}$} & Adecuada & $43(91,49 \%)$ \\
\hline & $\begin{array}{l}\text { No } \\
\text { adecuada }\end{array}$ & $4(8,51 \%)$ \\
\hline \multirow{2}{*}{$\begin{array}{l}\text { Programas para la elaboración de juegos } \\
\text { interactivos (para las actividades), como } \\
\text { Cerebriti }\end{array}$} & Adecuada & $45(95,74 \%)$ \\
\hline & $\begin{array}{l}\text { No } \\
\text { adecuada }\end{array}$ & $2(4,26 \%)$ \\
\hline \multirow{2}{*}{$\begin{array}{l}\text { Plataforma para el fomento de la lectura } \\
\text { como Ta-tum }\end{array}$} & Adecuada & $47(100 \%)$ \\
\hline & $\begin{array}{l}\text { No } \\
\text { adecuada }\end{array}$ & - \\
\hline \multirow{2}{*}{$\begin{array}{l}\text { Plataformas de alojamiento de vídeos } \\
\text { como Youtube }\end{array}$} & Adecuada & $23(48,94 \%)$ \\
\hline & $\begin{array}{l}\text { No } \\
\text { adecuada }\end{array}$ & $24(51,06 \%)$ \\
\hline \multirow{2}{*}{$\begin{array}{l}\text { Redes Sociales educativas como Edmodo } \\
\text { o Leoteca }\end{array}$} & Adecuada & $30(63,83 \%)$ \\
\hline & $\begin{array}{l}\text { No } \\
\text { adecuada }\end{array}$ & $17(36,17 \%)$ \\
\hline \multirow[t]{2}{*}{ Audiolibros o Podcast } & Adecuada & $34(72,34 \%)$ \\
\hline & $\begin{array}{l}\text { No } \\
\text { adecuada }\end{array}$ & $13(27,66 \%)$ \\
\hline
\end{tabular}

\section{DISCUSIÓN Y CONCLUSIONES}

El objetivo general de este estudio era recopilar información de la percepción que tenía la ciudadanía sobre la decisión de virtualizar la enseñanza por el confinamiento derivado del COVID-19. Teniendo en cuenta que este tipo de educación requiere niveles altos de autonomía y tesón, se tomó como referencia las etapas de Educación Infantil y Primaria, colectivo que puede ser más vulnerable por su edad y por requerir en mayor medida ayuda por parte de los habitantes del hogar para poder seguir este tipo de docencia. Los datos parecen indicar que esta medida se percibe de forma positiva entre la población no docente que indica que las TIC han permitido continuar con la educación de los más pequeños, pese a ello es destacable que la mayoría de los encuestados (más del $85 \%$ ) haya indicado que no consideran que se aprenda igual que en una clase presencial. Sin duda un dato que parece demostrar los beneficios que tiene una educación escolar como la que tenemos en España. En esta línea, nuestros resultados son similares a las opiniones reflejadas en el estudio de Díez y Gajardo (2020) donde se refe- 
rencia la necesidad humana del aprendizaje y la necesidad de la presencialidad para garantizar la igualdad de oportunidades.

Teniendo el cuenta que uno de los objetivos de la Agenda 2030 de la UNESCO (el Objetivo de Desarrollo Sostenible 4; ODS 4) es "Garantizar una educación inclusiva, equitativa y de calidad, y promover oportunidades de aprendizaje durante toda la vida para todas las personas", y en base a los hallazgos de la presente investigación, se concluye que si bien las TIC han permitido abordar de forma temporal una crisis educativa se debe seguir trabajando para que esta alternativa sea un modo realmente efectivo de seguir con la alfabetización en los casos en que la educación obligatoria no pueda realizarse en el centro escolar. En tal caso, se observa que la percepción global se inclina a pensar que el uso de este tipo de recursos para estudiantes menores de edad aumenta su vulnerabilidad y la brecha digital, considerando incluso que no se promueven las mismas oportunidades de aprendizaje para todos, aspecto que reafirma los hallazgos de García, et al., (2020). Tomando estos datos y las conclusiones de Díez y Gajardo (2020), se confirma que a día de hoy todavía se puede hablar de brecha digital en España y que estas condiciones además amplifican la brecha social, viéndose aumentadas ambas durante el confinamiento. Parece que a pesar de ser un país desarrollado, y de que la mayoría de hogares cuenta con los materiales tecnológicos básicos, la sensación que han tenido los ciudadanos en general y los estudiantes en particular es que les ha sido difícil seguir el curso académico o progresar en su aprendizaje, lo que nos lleva a concluir que las desigualdades digitales se ven influenciadas también por el aprovechamiento diferencial que los usuarios realizan de los recursos tecnológicos disponibles (Ragnedda, 2017, citado en Gómez, 2019). A pesar de ello, la pandemia ha reforzado el uso de las TIC ofreciendo la oportunidad de reformular la educación. Los recursos utilizados durante estos meses para acercar la docencia a casa han mostrado ser de gran utilidad y, como ha podido verse con las respuestas de los participantes de esta investigación, se consideran adecuadas herramientas que en la enseñanza presencial apenas se utilizan, confirmando así que se pueden transformar en valiosos recursos educativos.

El nivel de competencia digital se percibe también como condicionante de este tipo de enseñanzas. En este sentido se valora de forma más o menos similar a todos los agentes educativos implicados, ya que se destaca no solo condicionante el nivel de estudiantes o profesores sino también el nivel y uso de las TIC que puedan tener las familias o los supervisores de los menores. Los hallazgos de Díez y Gajardo (2020) mostraron que únicamente el 5,2\% de los encuestados (adultos a cargo del proceso educativo de los menores durante la pandemia) señaló tener disponibilidad para acompañarlos en su proceso educativo y que un 19,8\% se sentía poco o nada preparado. Si a estos datos sumamos que la mayoría de los encuestados en esta investigación (más del 87\%) señalaron que consideraban que la responsabilidad de las clases online recae sobre las familias, parece pertinente indicar que es necesario, y uno de los retos actuales, fomentar la mejora de las competencias y habilidades digitales. En este sentido, también apoyamos las conclusiones de Parody, Santos, Alcalá del Olmo e Isequilla (2019), quienes señalan que es fundamental seguir involucrando a las familias en el proceso de enseñanza-aprendizaje. En esta línea, y en consecuencia con las conclusiones de Domingo-Coscollola, Bosco-Paniagua, Carrasco-Segovia y Sánchez-Valero (2020), se observa la necesidad de una alfabetización digital y una mejora en la adquisición de competencias digitales, en relación con el uso pedagógico de la tecnología y una formación más práctica y experimental (Dias-Trindade, Moreira y Gomes, 2020) que vincule la sociedad con los centros educativos, incrementando la autonomía y la ciudadanía digital, contribuyendo así, no solo al desarrollo de habilidades necesarias para la vida y la formación sino también para romper las posibles barreras entre la familia y la escuela (Macià y Garreta, 2018).

Para finalizar, si bien es cierto que los resultados de esta investigación son consecuentes con los hallazgos de estudios similares, es imprescindible resaltar la principal limitación del estudio. En este sentido se hace pertinente realizar una investigación con una muestra mucho mayor que permita generalizar los datos de la ciudadanía no docente y conocer los recursos que se estiman más adecuados para abordar la estrategia que permita una mayor alfabetización digital y educativa. 


\section{PERCEPCIÓN CIUDADANA DEL USO DE LAS TIC Y LA ENSEÑANZA ONLINE DURANTE LA PANDEMIA}

\section{REFERENCIAS BIBLIOGRÁFICAS}

Álvarez-Sigüenza, J. (2019). Nativos digitales y brecha digital: Una visión comparativa en el uso de las TIC. Revista de la Asociación Española de Investigación de la Comunicación, 6(1), 203-223. https://doi.org/10.24137/raeic.6.11.12

Cohen, L., Manion, L. y Morrison K. (2010). Research methods in education. London: Routlegde.

Dias-Trindade, S., Moreira, J. A., \& Gomes, A. (2020). Assessment of University Teachers on their digital competences. Qwerty-Open and Interdisciplinary Journal of Technology, Culture and Education, 15(1), 50 69. DOI: $10.30557 /$ QW000025

Díez, E., \& Gajardo, K. (2020). Educar y evaluar en tiempos de Coronavirus: la situación en España. Multidisciplinary Journal of Educational Research, 10(2), 102-134. doi:http://dx.doi.org/10.17583/remie.2020.5604

Domingo-Coscollola, M., Bosco-Paniagua, A., Carrasco-Segovia, S., \& Sánchez-Valero, J. A. (2020). Fomentando la competencia digital docente en la universidad: Percepción de estudiantes y docentes. Revista de Investigación Educativa, 38(1), 167-182. D0l: https://doi.org/10.6018/rie.340551

García, N., Rivero, M.L., y Ricis, J. (2020). Brecha digital en tiempo del Covid-19. Revista Educativa Hekademos, 28, 76-85. Recuperado a partir de https://www.hekademos.com/index.php/hekademos/article/view/9

Gómez, D. (2019). Una aproximación a la evolución de la brecha digital entre la población joven en España (2006-2015). RES. Revista Española de Sociología, (28), 27-44. D0l:10.22325/fes/res.2018.16

Hargittai, E. (2002). Second-Level Digital Divide: Differences in People's Online Skills. First Monday, 7(4), 1-14. D0l: https://doi.org/10.5210/fm.v7i4.942

I.N.E. (2019). Encuesta sobre Equipamiento y uso de Tecnologías de Información y Comunicación en los hogares. INE.

López, M. (2018). La educación como derecho fundamental y como servicio público en España. En Servicios de interés general y protección de los usuarios (pp. 109-129). Madrid, España: Dykinson.

Macià, M., \& Garreta, J. (2018). Accesibilidad y alfabetización digital: barreras para la integración de las TIC en la comunicación familia/escuela. Revista de Investigación Educativa, 36(1), 239-257. D0I: https://doi.org/10.6018/rie.36.1.290111

Pastran, M., Gil, N. A., \& Cervantes, D. (2020). En tiempos de coronavirus: las TIC S son una buena alternativa para la educación remota. Revista Boletín Redipe, 9(8), 158-165. https://doi.org/10.36260/rbr.v9i8.1048

Parody, L. M., Santos, M. J., Alcalá del OImo, M. J., \& Isequilla, E. (2019). El desafío educativo del siglo XXI: Relevancia de la cooperación entre familia y escuela. ECP. Espiral. Cuadernos del Profesorado. Revista multidisiplinar de educación, 12(24), 19-29. http://hdl.handle.net/10835/7295

Rodicio-García, M. L., Ríos-de Deus, M. P., Mosquera-González, M. J., \& Penado, M. (2020). La brecha digital en estudiantes españoles ante la Crisis de la Covid-19. Revista Internacional de Educación para la Justicia Social, 9(3), 103-125. https://doi.org/10.15366/riejs2020.9.3.006

Sierra, C. A. (2013). La educación virtual como favorecedora del aprendizaje autónomo. Panorama, 5(9), 75-87. https://doi.org/10.15765/pnrm.v5i9.37

Torres, C. (2017). Sociedad de la información y brecha digital en España. Panorama Social, (25), 17-33. Recuperado de https://dialnet.unirioja.es/servlet/articulo?codigo=6371386 


\author{
Anexo1: \\ Cuestionario de investigación.
}

El siguiente cuestionario corresponde a una investigación empírica sobre los posibles efectos que tuvo el cierre de los centros educativos y las clases online en la alfabetización de los estudiantes de Educación Infantil y Primaria.

Tu colaboración podrá contribuir a ampliar y mejorar el conocimiento científico relativo a este estudio. Por lo tanto, te rogamos que completes el cuestionario prestando especial atención a no dejar ninguna pregunta sin responder. No te llevará más de un par de minutos y es importante para nosotros que contestes esta pequeña encuesta.

La información aquí proporcionada es anónima y será utilizada de forma general y no a nivel individual. Así mismo el tratamiento de la información está sujeto a la Ley Orgánica 3/2018, de 5 de diciembre, de Protección de Datos Personales y garantía de los derechos digitales.

Con tu participación harás que la ciencia avance ¡Muchas gracias por tu colaboración!

\title{
1) Datos sociodemográficos
}

\section{Edad, Sexo, Nacionalidad, CCAA}

1.a Preguntas comunes

-¿Has tenido hijos o personas a cargo durante las clases online por el cierre de centros educativos?

- ¿Trabajabas fuera de casa durante el confinamiento?

-¿Eras profesor/a o una de las personas que impartía clases online (a Infantil o Primaria) durante el confinamiento?

\section{2) Preguntas de investigación (escala dicotómica)}

El cierre de los centros educativos de Educación Infantil y Primaria ha provocado que muchos utilizasen las TIC para continuar con la enseñanza online, en este sentido:

- Considero que la utilización de las TIC para continuar con la enseñanza (en modalidad online) era lo único que se podía hacer.

- Considero que los estudiantes han aprendido lo mismo con la enseñanza online que si hubiesen acudido a clase de forma presencial.

- Considero que la utilización de las TIC para continuar con la enseñanza (en modalidad online) promueve las mismas oportunidades de aprendizaje para todos los estudiantes)

- Considero que el cierre de los centros educativos ha incrementado la brecha digital entre los estudiantes (desigualdad en el acceso a Internet, a los dispositivos digitales, etc.) (si o no)

- Considero que el cierre de los centros educativos aumenta la vulnerabilidad de los estudiantes (al ser menores de edad)

- Considero que los conocimientos que los tutores, padres o supervisores de los estudiantes pueden tener del uso de las TIC puede condicionar la efectividad de las clases online

- Considero que la impartición de clases online ha permitido la alfabetización de los estudiantes de Educación Infantil y Primaria.

- Considero que la alfabetización de los estudiantes durante la pandemia ha estado condicionada por el nivel de competencia digital que tuviese el docente.

- Considero que la alfabetización de los estudiantes durante la pandemia ha estado condicionada por el nivel de competencia digital que tuvieran los estudiantes.

- Considero que con las clases online la responsabilidad educativa recayó sobre las familias. 


\section{3) Recursos educativos según etapas}

3.a Una vez que los centros comenzaron con las clases online ¿qué recurso consideras más apropiado para Educación Infantil?:

a) Videoconferencias o clases del profesor en directo

b) Uso de juegos educativos

c) Uso de vídeos educativos (Video-Lección)

d) Fichas y lecturas para hacer en casa

e) Otro

3.b Una vez que los centros comenzaron con las clases online ¿qué recurso consideras más apropiado para Educación Primaria?

a) Videoconferencias o clases del profesor en directo

b) Uso de juegos educativos

c) Uso de vídeos educativos (Video-Lección)

d) Fichas y lecturas para hacer en casa

e) Actividades y ejercicios del manual de la asignatura

f) Uso de blog y Redes Sociales educativas

g) Elaboración de trabajos y presentaciones para exponer a distancia

h) Otro

4. Adecuación de herramientas digitales

Para finalizar, indique, según su opinión el grado de adecuación de las siguientes herramientas utilizadas para para poder impartir sus clases de forma online durante la pandemia:

Herramientas de videoconferencias como Zoom

> Programas para la elaboración de presentaciones como Genial.ly o Prezi

> Programas para la elaboración de juegos interactivos (para las actividades), como Cerebriti

$>$ Plataforma para el fomento de la lectura como Ta-tum

$>$ Plataformas de alojamiento de vídeos como Youtube

$>$ Redes Sociales educativas como Edmodo o Leoteca

$>$ Audiolibros o Podcast

¡Muchas gracias por la colaboración! 